\title{
Neuropsychological Profile of Treated Children with Congenital Toxoplasmosis
}

\author{
Kimberly A. Kerns ${ }^{1 *}$, Sandra J. Mish ${ }^{1}$, Jillian Roberts², Frank Jagdis ${ }^{3,4}$ \\ ${ }^{1}$ Department of Psychology, University of Victoria, Victoria, Canada \\ ${ }^{2}$ Educational Psychology and Leadership Studies, University of Victoria, Victoria, Canada \\ ${ }^{3}$ University of British Columbia, Vancouver, Canada \\ ${ }^{4}$ Queen Alexandra Centre for Children's Health, Victoria, Canada \\ Email: ${ }^{*}$ kkerns@uvic.ca
}

Received 1 May 2014; revised 28 May 2014; accepted 21 June 2014

Copyright (C) 2014 by authors and Scientific Research Publishing Inc. This work is licensed under the Creative Commons Attribution International License (CC BY). http://creativecommons.org/licenses/by/4.0/

\section{(c) (i) Open Access}

\begin{abstract}
Background: Very little is known about the long-term neuropsychological outcomes of infants who were treated with pyrimethamine and sulfadiazine for congenital Toxoplasma infection. This study investigated the neurocognitive functioning and behavior of a cohort of treated infants exposed to Toxoplasma gondii in the 1994-1995 outbreak in Victoria, British Columbia. Methods: Ten infants from the original cohort, treated with pyrimethamine and sulfadiazine starting during first year of life and continuing for 12 months, were evaluated at 7 to 8.5 years of age. Neuropsychological evaluations focused on the cognitive, academic, adaptive, and behavioral functioning of these children. Results: The cohort demonstrated generally average neurocognitive abilities and academic achievement. According to parental ratings, the children's executive functions, behavior and adaptive functioning did not differ significantly from the normative population. However, subtle difficulties were seen on more demanding sustained attention and impulse control tasks. Conclusions: These results suggest favorable outcomes in infants who were treated for one year with pyrimethamine and sulfadiazine. Nonetheless, congenital toxoplasmosis might contribute to some difficulties with higher-order cognitive abilities or tasks demanding greater self-monitoring. These difficulties could become more pronounced as higher demands for these abilities and brain areas come online during late childhood and adolescence. These findings highlight the importance of effective treatment and longitudinal follow-up of children with congenital toxoplasmosis.
\end{abstract}

\section{Keywords}

Toxoplasmosis, Congenital Infection, Neuropsychological Effects, Neurocognitive Outcome, Child

\footnotetext{
${ }^{*}$ Corresponding author.
} 


\section{Background}

Congenital toxoplasmosis is a disease caused by the protozoan parasite, Toxoplasma gondii (T. gondii). *Toxoplasma infections occur worldwide, although widely disparate prevalence rates exist throughout the world depending on geographic region and sanitation practices. The incidence is difficult to evaluate in North America because toxoplasmosis is not generally a reportable disease, and prenatal screening programs for T. gondii infection do not routinely occur (Canadian Paediatric Society, 1999; Remington, McLeod, Thulliez, \& Desmonts, 2006). Congenital toxoplasmosis is believed to affect approximately 1 out of every 10,000 live births in the United States each year (Guerina, Hsu, Meissner, Maguire, Lynfield, Stechenberg et al., 1994). Although the incidence of congenital infection is low, sequelae associated with this disease are of significant clinical importance.

Congenital toxoplasmosis results mostly after a primary maternal infection with $T$. gondii during pregnancy and subsequent transplacental transmission of the parasite to the developing fetus. Gestational age at the time of maternal infection is a determinant of intrauterine infection and severity of congenital disease. Maternal-fetal transmission rate is highest during the third trimester of pregnancy (Dunn, Wallon, Peyron, Peterson, Peckham, \& Gilbert, 1999; Foulon, Villena, Stray-Pederson, Decaster, Lappalainen, Pinon et al., 1999; Remington et al., 2006). Nonetheless, a late maternal infection often results in subclinical or mild fetal infection (Desmonts \& Couvreur, 1974; Remington et al., 2006). Although maternal infection is less frequently transmitted to the fetus early in pregnancy, early transmission is often associated with fetal death or severe congenital disease (Desmonts \& Couvreur, 1974). Antiparasitic treatment during pregnancy often reduces the severity of sequelae in infected infants (Foulon et al., 1999; Daffos, Forestier, Capella-Pavlovsky, Thulliez, Aufrant, Valenti, \& Cox, 1988; Hohlfeld, Daffos, Thulliez, Aufront, Couvreur, MacAlees et al., 1989). However, current medications do not impact maternal-fetal transmission rates (Foulon et al., 1999; Gilbert, Gras, Wallon, Peyron, Ades, \& Dunn, 2001).

Toxoplasma gondii has been shown to invade many different tissues in the body, including the brain, skeletal and cardiac muscle, liver, retina, and choroid (Derouin, 2001). After an acute congenital infection, Toxoplasma cysts may persist in the eye and central nervous system (CNS). Treatment is ineffective at eradicating the encysted form of the parasite (Derouin, 2001). It is believed that infected individuals harbor these tissue cysts for life (latent infection), and cysts that rupture in later life could lead to tissue damage (Remington et al., 2006).

A wide spectrum of clinical manifestations is associated with congenital toxoplasmosis, which may result in neurological, systemic, audiological, and ocular abnormalities. The classic clinical triad of congenital Toxoplas$m a$ infection includes hydrocephalus, intracranial calcifications, and chorioretinitis, although it is unusual for an infected infant to show all three signs. Chorioretinitis is the most common clinical feature of this disease. Congenital toxoplasmosis often goes undiagnosed in the neonatal period as the majority of infants (85\% - 90\%) have no obvious signs or symptoms of the infection on routine medical examinations (Remington et al., 2006). Infants with subclinical disease, if left untreated or treated for less than one month, will often develop adverse sequelae over time (Couvreur \& Desmonts, 1962; Eichenwald, 1960; Koppe, Loewer-Sieger, \& de Roever-Bonnet, 1986; Mets, Holfels, Boyer, Swisher, Roizen, Stein et al., 1996; Wilson, Remington, Stagno, \& Reynolds, 1980). Delayed sequelae can include impaired vision or blindness from chorioretinitis, seizures, motor deficits, hearing loss, mental retardation and/or lowered intellectual scores over time.

Early diagnosis and prompt initiation of intensive antiparasitic treatment (usually for one year) is associated with more favorable neurodevelopmental, ophthalmologic, and audiologic outcomes, even for many children with substantial involvement at birth (Guerina et al., 1994; Mets et al., 1996; McLeod, Boyer, Karrison, Kasza, Swisher, Roizen et al., 2006; Patel, Holfels, Vogel, Boyer, Mets, \& Swisher, 1996; Roizen, Swisher, Stein, Hopkins, Boyer, Holfels et al., 1995; McAuley, Boyer, Patel, Mets, Swisher, Roizen et al., 1994; Wallon, Kodjikian, Binquet, Gerweg, Fleury, Quantin, Peyron et al., 2004; Brézin, Thulliez, Couvreur, Nobré, McLeod, \& Mets, 2003; Peyron, Wallon, \& Bernardoux, 1996; McGee, Wolters, Stein, Kraus, Johnson, Boyer et al., 1992). The National Collaborative Chicago-Based, Congenital Toxoplasmosis Study (McLeod et al., 2006) found more favorable outcomes in the majority of children treated for 1 year with pyrimethamine, sulfadiazine, and leucovorin than previously reported for children who were untreated or treated for 1 month. However residual deficits may develop even after early intensive treatment. Visual impairments may result from reactivation of the latent tissue cysts, which cause recurrent active chorioretinitis or new lesions during childhood or even adulthood (Remington et al., 2006; Mets et al., 1996; McLeod et al., 2006; Wallon et al., 2004; Peyron et al., 
1996).

Few outcome studies of treated children with congenital Toxoplasma infection have evaluated intellectual abilities. In a series of studies completed by The Chicago Collaborative Treatment Trial, normal intellectual abilities were found in many of the treated children, with intelligence quotient (IQ) scores ranging from 85 to 140 (McAuley et al., 1994). Lower intellectual scores were noted in some treated children as compared to their uninfected siblings, although repeat testing did not reveal a significant decline in intellectual abilities over time (Roizen et al., 1995). A follow-up study by the National Collaborative Chicago-Based, Congenital Toxoplasmosis Study (McLeod et al., 2006) found normal intellectual abilities in 77\% of treated children with varying degrees of disease involvement at birth. All of the children with no or mild disease had IQ scores greater than 70; however, one child showed a decrease in greater than 15 IQ points over two consecutive evaluations. For children who had moderate to severe neurological disease at birth, 73\% scored greater than $70 \%$ on intellectual testing and $84 \%$ did not show a significant decline in intellectual abilities over time (IQ $\geq 15$ ).

Koppe et al. (1986) found no difference in school performance between children with congenital toxoplasmosis and age-matched peers, although these authors did not differentiate between treated and untreated children. In addition, standardized measures were not used to assess academic achievement in this study.

A questionnaire study at diverse European centers reported parental observations on outcomes of their child at three years of age (Freeman, Salt, Prusa, Malm, Ferret, Buffolano et al., 2005). There was no evidence of impaired development or behavior in treated children with congenital toxoplasmosis as compared to age-matched controls. However, systematic assessment of functioning at later ages is lacking in children with treated congenital toxoplasmosis.

Little is known about the long-term prognosis of treated infants, nor the impact of treatment on academic achievement, more specific neuropsychological functioning (e.g., attention, memory, language, visuomotor skills, executive functions), and behavior across development. Pediatricians, psychologists and education professionals may need to monitor these children for evidence of relapses and late-onset problems, as congenital disease may continue to have an impact on specific organ function, cognitive development and school performance.

\section{Description of Current Study}

The current study was part of a comprehensive research project investigating the long-term outcomes of a unique cohort of children who received similar treatment regimens after being exposed to a unique clonal type of T. gondii in utero during a 1994-1995 waterborne outbreak in Victoria, British Columbia. The purpose of the present study was to extend our knowledge about the long-term outcome of treatment for congenital toxoplasmosis, and to provide a comprehensive evaluation of the neuropsychological functioning, academic achievement and behavior of this unique cohort of treated children. A previous article based on this same sample of children focused on the contextual, familial impact of congenital toxoplasmosis on the parents, and recommendations and insights provided by the families of these children from qualitative interviews were summarized (Roberts, Mortimer, Mish, Kerns, Jagdis, \& MacMath, 2005). To our knowledge, this is the first study to systematically evaluate the neuropsychological functioning of treated children with congenital toxoplasmosis.

\section{Methods}

\subsection{Description of Outbreak}

The toxoplasmosis outbreak in the Greater Victoria region was suspected when health professionals noted an increase in serologically positive cases of acute Toxoplasma infection. One hundred acute cases between the ages of 6 and 83 were identified. The municipal drinking water supply was felt to be the source of the organism because of the distribution of cases in the geographic area and the absence of a common identified source in case control studies. At the time, it was the first recorded outbreak to be associated with a municipal drinking water supply (Bowie, King, Werker Isaac-Renton, Bell, Eng, \& Marion, 1997).

\subsection{History of Diagnosis and Treatment}

Following recognition of the outbreak, a massive screening program for pregnant and postnatal women was undertaken. Screening was offered to women who were pregnant at any time between October 1, 1994 and June 30 , 
1996. Based on this screening, 36 of the 3812 screened women tested positive for acute acquired T. gondii infection. A diagnosis of toxoplasmosis was confirmed based on serological findings as described elsewhere (Bowie et al., 1997). Prenatal treatment was initiated once maternal infection was diagnosed, and continued until the end of pregnancy. Of the infants identified with congenital toxoplasmosis, only 4 mothers were treated prenatally with spiramycin. The mean duration of prenatal treatment was 27.75 days (range, 5 - 75 days).

All 36 infants underwent laboratory confirmation of congenital toxoplasmosis. They were tested for IgM, IgG and IgA antibodies to T. gondii, as well as the Dye Test. Twelve infants were identified with congenital toxoplasmosis, and completed a one-year treatment regimen of pyrimethamine, sulfadiazine, and leucovorin according to the recommended treatment protocol for congenital toxoplasmosis (Remington et al., 2006), with weekly monitoring of blood counts. Mean age at treatment initiation was 86.8 days (range, birth to 274 days). Five children started treatment within the first 3 weeks of life. Specialists in pediatric infectious diseases coordinated evaluation and treatment of the infants.

\subsection{Participants}

The investigated children were recruited from the original cohort of 12 infants infected in the British Columbia toxoplasmosis outbreak (Bowie et al., 1997). At follow-up in 2001-2002, 10 children (6 males and 4 females) were available for neuropsychological evaluation. All children were between 7 and 8.5 years of age. One family could not be located, and one declined evaluation. Eight families were still residing in the Greater Victoria area, while the others were residing outside the area but were willing to return for testing.

\subsection{Medical History}

Neurological and ophthalmologic sequelae are summarized in Table 1. During infancy, 3 of the 10 children with congenital toxoplasmosis demonstrated CNS abnormalities. On brain imaging, 2 had intracranial calcifications; one of which was widespread. One case had features of meningoencephalitis on cerebrospinal fluid analysis. However, six children had been diagnosed with chorioretinitis, and all the children were continuing to be followed for further ophthalmologic assessment due to the continued risk for this condition.

Table 1. Summary of neurological and ophthalmologic signs and symptoms.

\begin{tabular}{|c|c|}
\hline Signs and Symptoms & Children Affected, No. $(\mathrm{n}=10)$ \\
\hline \multicolumn{2}{|l|}{ Neurological $^{\mathrm{a}}$} \\
\hline - $\quad$ No findings & 7 \\
\hline \multicolumn{2}{|l|}{ - Major findings } \\
\hline ○ Hydrocephalus & 0 \\
\hline$\circ$ Intracranial calcifications & 2 \\
\hline$\circ \quad$ Meningoencephalitis & 1 \\
\hline \multicolumn{2}{|l|}{ Ophthalmologic } \\
\hline - No ocular involvement & 4 \\
\hline - Chorioretinitis $^{\mathrm{b}}$ & 6 \\
\hline \multicolumn{2}{|l|}{ Bilateral } \\
\hline ○ Visual impairment & 2 \\
\hline$\circ$ Minimal to no impairment & 2 \\
\hline \multicolumn{2}{|l|}{ Unilateral } \\
\hline$\circ$ Visual impairment & 0 \\
\hline$\circ$ Minimal to no impairment & 2 \\
\hline - Recurrence or new chorioretinitis ${ }^{\mathrm{c}}$ & 4 \\
\hline \multicolumn{2}{|l|}{ Other abnormality } \\
\hline - Hearing loss & 0 \\
\hline - $\quad$ Precocious puberty & 1 \\
\hline
\end{tabular}

${ }^{\mathrm{a}}$ No abnormalities on routine newborn physical evaluations. Neurological signs were identified; t time of diagnosis and initiation of treatment. ${ }^{\mathrm{b}}$ Five children received diagnosis of chorioretinitis; between 2 weeks and 7 months; one child had onset of new retinal lesions at age 5 and 9 . ${ }^{\text {B Based on }}$ medical reports up to and including May 2004. 


\subsection{Procedure and Measures}

The research protocol was approved by the University of Victoria Human Research Ethics Board and the Vancouver Island Health Authority Child, Youth and Maternal Health Program Research Advisory Committee. Informed written consent and verbal assent were obtained from parents and children, respectively, prior to any testing or interviews. Each family received \$50 for participating in the study.

Children were tested in two sessions at the University of Victoria. Intellectual and academic achievement tests were individually administered along with a broad-based battery of neuropsychological function and behavior selected to provide a neuropsychological profile for each child. A full list of the standardized and experimental measures is included in Appendix A. The test battery assessed ten major areas of functioning, including general intelligence, achievement, motor skills, visuomotor skills, language, attention, memory, executive functions, behavior, and adaptive skills. Children who participated in the study did not exhibit any hearing loss or other severe clinical sequelae (e.g., blindness) that would preclude an accurate evaluation.

Parents completed questionnaires regarding their perceptions of their children's executive functions, behavior, and adaptive functioning (Appendix A), as well as participating in a qualitative interview about familial experiences and recommendations. A debriefing of testing results and a brief written report of individual findings were provided to all participating parents.

Additionally, all children underwent a medical examination by a pediatric physician with a specialization in infectious diseases or by their family physician. A follow-up history was obtained at the time of examination.

\subsection{Data Analysis}

Neuropsychological test scores were compared with means of the normative populations on which the tests were standardized (using appropriate normative data for the age and gender of the child) and were converted into standardized scores. This allowed us to quantify differences in the mean test scores of this group of children compared with the normative population using SD from the mean of the latter group. Next, to reduce the number of potential comparisons, tests were grouped by the major cognitive domains, as illustrated in Table 2. An overall or "grand average" was created by averaging the scores which were all standardized and converted to zscores. These domain averages were then analyzed using an absolute test [via t-test procedure of the Statistical Package for Social Sciences (SPSS)], to assess the likelihood that the group mean differed significantly from the anticipated "average” level and statistically comparing this sample against anticipated scores based on a typically developing normative population (Jeremy, Kim, Nozyce, Nachman, McIntosh, Pelton et al., 2005).

Table 3 summarizes children's performance on measures of attention and executive function, grouped according to subdomains due to the multifactorial nature of these cognitive processes. Subdomains included rote

Table 2. Broad cognitive domains and measures.

\begin{tabular}{|c|c|}
\hline Cognitive Domain & Measures \\
\hline \multicolumn{2}{|l|}{ Intellectual ability } \\
\hline Verbal & WISC-III, Verbal Comprehension Index (VCI) \\
\hline Nonverbal & WISC-III, Performance Organization Index (POI) \\
\hline Academic achievement & WJ-III ACH, Total Achievement \\
\hline Motor speed & $\begin{array}{l}\text { Grooved Pegboard, Dominant \& Nondominant Hands } \\
\text { NEPSY, Manual Motor Sequencing }\end{array}$ \\
\hline Visuomotor skills & $\begin{array}{l}\text { RCFT, Copy } \\
\text { NEPSY, Design Copying \& Visuomotor Precision } \\
\text { WISC-III, Coding }\end{array}$ \\
\hline Language & NEPSY, Phonological Processing \& Verbal Comprehension \\
\hline Verbal memory & WRAML, Stories \& Verbal Learning (Immediate \& Delayed) \\
\hline Visual memory & WRAML, Picture Memory, Design Memory \& Visual Learning (Immediate \& Delayed) \\
\hline
\end{tabular}

Note: WISC-III, Wechsler Intelligence Scale for Children Third Edition; WJ-III ACH, Woodcock-Johnson III Tests of Achievement; NEPSY, A Developmental Assessment; RCFT, Rey Complex Figure Test; WRAML, Wide Range Assessment of Memory and Learning. 
Table 3. Subdomains of attention and executive control.

\begin{tabular}{cc}
\hline Outcome Subdomain & Measures Included \\
\hline Rote Repetition & WISC-III, Digit Span Forwards \\
WRAML, Sentence Memory \\
Selective Attention & WISC-III, Symbol Search \\
NEPSY, Visual Attention \\
Sustained Attention & TEA-Ch, Code Transmission \\
Working Memory & CPT, Hit RT SE \& Hit RT ISI Change \\
Impulse Control & WISC-III, Digit Span Backwards \\
CSOT \\
Dual Tasking & TEA-Ch, Walk, Don't Walk \\
Fluency & Fire Fighter Game, SSRT \\
Parent Rating of Executive Control & TEA-Ch, Creature Counting \& Opposite Worlds \\
\end{tabular}

Note: WISC-III, Wechsler Intelligence Scale for Children Third Edition; WRAML, Wide Range Assessment of Memory and Learning; NEPSY, A Developmental Assessment; TEA-Ch, Test of Everyday Attention for Children; CPT, Conners' Continuous Performance Test; Hit RT SE, Hit Rate Standard Error; Hit RT ISI Change, Hit Rate Inter-Stimulus Interval Change; CSOT, Children's Size-Ordering Task; SSRT, Stop Signal Reaction Time; BRIEF, Behavior Rating Inventory of Executive Function.

repetition, selective attention, sustained attention, working memory, impulse control, dual tasking, and fluency. Results were also analyzed in this manner for the Behavior Rating Inventory of Executive Function (BRIEF) Global Executive Composite, representing parent report of executive function.

\section{Results}

As a group, this sample of children with congenital toxoplasmosis exhibited IQ scores that statistically were slightly above an average of 100 . Overall, this group of children performed within the average range on measures of verbal (WISC-III Verbal Comprehension Index (VCI): $\mathrm{M}=109.6, \mathrm{SD}=9.95)$ and nonverbal reasoning (WISC-III Perceptual Organization Index (POI): $\mathrm{M}=109.4$, SD = 11.51). Their scores on measures of academic achievement, motor, visual-motor, language and both verbal and visual memory functions were also within the average range (Figure 1).

The children with congenital toxoplasmosis also scored within normal limits on the majority of subdomains of attention and executive function. Parental ratings of executive function on the BRIEF were also within age level expectations. However, statistical analyses revealed that on neuropsychological tests of sustained attention and impulse control as a group they scored significantly lower than anticipated (Figure 2).

Overall, the children's mean scores on the Conner's Parent Rating Scales did not indicate that as a group there were any psychosocial problems (i.e., where T-scores $>65$ indicate clinically significant problems). However, 3 of 10 children with congenital toxoplasmosis did demonstrate significant elevations on more than five behavioural scales, and one child showed a significant elevation on the psychosomatic subscale (Table 4). Overall, the group also demonstrated age-appropriate adaptive functioning.

\section{Discussion}

This study explored the neuropsychological profile of treated children exposed to toxoplasmosis in utero. Current results support prior research findings that infants treated with a standardized treatment of pyrimethamine and sulfadiazine for congenital toxoplasmosis have substantially better outcomes than infants with brief or no treatment.

The study provides preliminary evidence of generally average cognitive abilities and academic achievement in treated children with congenital toxoplasmosis. In fact, overall intellectual skills in this group of children were slightly above the average IQ of 100 . Nonetheless, on measures of attention and executive function there 


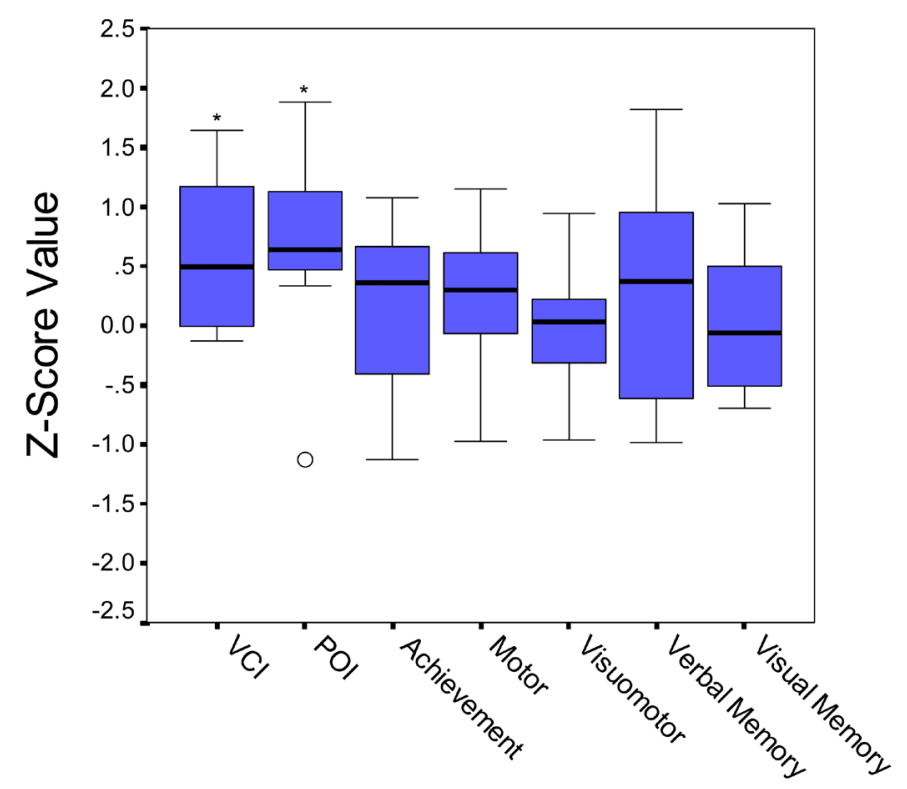

Figure 1. Boxplot. Performance on cognitive and academic measures based on established age-matched norms. Middle line in box represents median value; lower and upper edges of box represent $25^{\text {th }}$ and $75^{\text {th }}$ percentile of data set; and whiskers represent range of data values. $\circ$ indicates outlier. ${ }^{*} p<.05$.

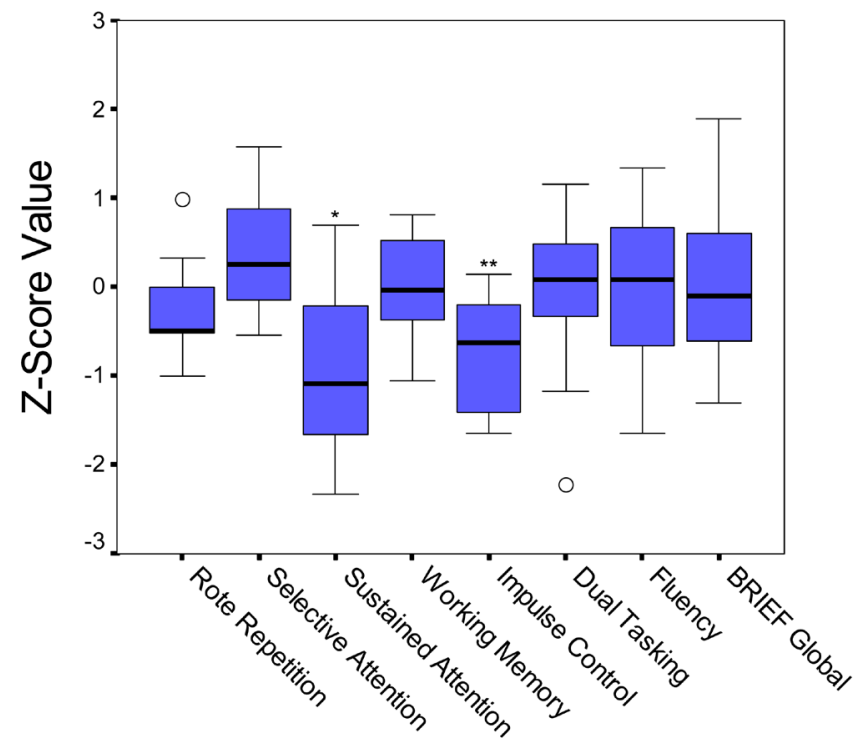

Figure 2. Boxplot. Performance on attention and executive function measures based on established age-matched norms. Middle line in box represents median value; lower and upper edges of box represent $25^{\text {th }}$ and $75^{\text {th }}$ percentile of data set; and whiskers represent range of data values. $\circ$ indicates outlier. ${ }^{*} p<.05 ;{ }^{* * *} p<.01$.

was some suggestion that congenital toxoplasmosis might contribute to subtle difficulties. Most notably, these children scored significantly lower than would be expected on measures of sustained attention and impulse control. As a group, their median score on sustained attention fell at only the $14^{\text {th }}$ percentile and on impulse control at the $27^{\text {th }}$ percentile versus the anticipated group average of $50^{\text {th }}$ percentile (average). It is important to note that there was considerable variation within the sample of children, with some exhibiting no difficulty on any of the cognitive tasks. However, the only measures on which participants scored in the clinically significant range were on tasks within the attention and executive function domains. 
Table 4. Clinical elevations on Conners’ parent rating scale.

\begin{tabular}{cc} 
Subscale & Children, No. (n = 10) \\
Psychosomatic & 3 \\
DSM-IV: Hyperactive-Impulsive & 3 \\
Cognitive Problems/Inattention & 2 \\
Hyperactivity & 2 \\
Conners' ADHD Index & 2 \\
Conners' Global Index: Restless-Impulsive & 2 \\
Conners' Global Index: Total & 2 \\
DSM-IV: Inattentive & 2 \\
DSM-IV: Total & 2 \\
Oppositional & 2 \\
Anxious-Shy & 1 \\
Conners' Global Index: Emotional Lability & 1 \\
\hline
\end{tabular}

Note: DSM-IV, Diagnostic and Statistical Manual of Mental Disorders—Fourth Edition; ADHD, Attention-Deficit/Hyperactivity Disorder.

\subsection{Limitations}

Our study has limitations that must be considered in interpreting the data. A major limitation of the study is the small sample size of only 10 participants. While this is a small sample size, it does represent the majority of the children exposed to $T$. gondii during the outbreak. Regardless, a larger sample would be optimal for more robust statistical analyses and allow for greater generalization to children with early treatment for exposure to T. gondii.

The representativeness of the sample is also limited. The present cohort was exposed to a unique strain of $T$. gondii during a community-wide outbreak linked to municipal drinking water. Given the early identification and treatment, these children did not present with severe manifestations of congenital infection. Caution is advised in generalizing the findings to other children with congenital toxoplasmosis. Our results should be viewed as specific to the treatment regimen followed in this study, not all treatment regimens for congenital toxoplasmosis.

Finally, a control group matched for specific characteristics to this sample would have been helpful, such as siblings. While we originally attempted to recruit sibling controls for all participants, there were an insufficient number with siblings. The group was therefore compared test by test to a large normative sample of typically developing children. These limitations need to be kept in mind when interpreting these results.

\subsection{Conclusions and Future Directions}

Notwithstanding the above limitations, the results of this preliminary study suggest age-appropriate cognitive, academic and behavioral functioning in treated children with subclinical congenital toxoplasmosis or minor early clinical signs. Participants presented with subtle clinically and statistically significant reduction in sustained attention and impulse control. Continued follow-up is indicated to confirm these initial results, and to exclude further long-term difficulties in higher order cognitive abilities.

At the time of testing, children in this cohort ranged from 7 to 8.5 years of age. Between the ages of 6 and 12 , children undergo considerable development within the domains of executive function, self-regulation and aspects of attention. The subtle differences this group of children exhibits, in comparison to typically developing children's average score, may represent a delay in the maturation of these abilities or could potentially highlight what might be a more enduring difficulty in this area. Thus, continued monitoring and longitudinal multidisciplinary follow-up of this population is warranted.

There is a need to better understand the developmental implications of congenital toxoplasmosis. As brain areas subserving complex attention and executive functions become prominent and important during late child- 
hood and adolescence when higher levels of self-regulation are anticipated, weaknesses may become more pronounced with age. It is important to determine whether the subtle deficits observed in this population represent a mild "delay" in development of these higher order cognitive abilities or herald a long-term difficulty in this area.

This research project used a blend of quantitative and qualitative methodology to provide a comprehensive understanding of the neurodevelopmental course and familial experiences of congenital toxoplasmosis. A thorough review of the contextual, familial impact of congenital toxoplasmosis on the parents has been previously published (Roberts et al., 2005). It is important to note, although the children presented with only minor physical and neuropsychological consequences, their parents continued to experience a great deal of concern regarding their child's future health. The lack of information with respect to the long-term effects of congenital toxoplasmosis, specifically in treated children, reinforces their fears of uncertainty about reoccurrence of symptoms in the future. This is consistent with other families with children diagnosed with a chronic illness (Katz, 2002; Dodgson, Garwick, Blozia, Patterson, Bennett, \& Blum, 2000). These findings underscore the importance of effective treatment, family-centered practice, and longitudinal follow-up.

\section{Acknowledgements}

The authors gratefully acknowledge the children and their families for their generous cooperation in this research. Appreciation is also extended to Jennifer A. Michel, MS, Robert J. McInerney, MS, and Tamara Mortimer, MA, for their contribution to data collection, and Andrew J. Burnett, MD, for his insights and ophthalmologic follow-up with the children.

\section{Financial Support}

This work was supported by a grant from the Queen Alexandra Foundation for Children, Victoria, British Columbia.

\section{Potential Conflicts of Interest}

All authors: no conflicts.

\section{References}

Bowie, W. R., King, A. S., Werker, D. H., Isaac-Renton, J. L., Bell, A., Eng, S. B., \& Marian, S. A. (1997). Outbreak of Toxoplasmosis Associated with Municipal Drinking Water. Lancet, 350, 173-177. http://dx.doi.org/10.1016/S0140-6736(96)11105-3

Brézin, A. P., Thulliez, P., Couvreur, J., Nobré, R., McLeod, R., \& Mets, M. B. (2003). Ophthalmic Outcomes after Prenatal and Postnatal Treatment of Congenital Toxoplasmosis. American Journal of Ophthalmology, 135, 779-784. http://dx.doi.org/10.1016/S0002-9394(02)01704-X

Bruininks, R. H., Woodcock, R. W., Weatherman, R. F., \& Hill, B. K. (1996). Scales of Independent Behavior-Revised Comprehensive Manual. Itasca, IL: Riverside Publishing.

Canadian Paediatric Society (1999). Common Questions about the Diagnosis and Management of Congenital Toxoplasmosis. Paediatrics \& Child Health, 4, 137-141.

Conners, C. K. (1994). Conners' Continuous Performance Test Computer Program 3.0 User's Guide. Toronto, ON: MultiHealth Systems Inc.

Conners, C. K. (1997). Conner's Rating Scales—Revised Technical Manual. Toronto, ON: Multi-Health Systems Inc.

Couvreur, J., \& Desmonts, G. (1962). Congenital and Maternal Toxoplasmosis: A Review of 300 Congenital Cases. Developmental Medical Child Neurology, 4, 519-530. http://dx.doi.org/10.1111/j.1469-8749.1962.tb03221.x

Daffos, F., Forestier, F., Capella-Pavlovsky, M., Thulliez, P., Aufrant, C., Valenti, D., Cox, W. L. et al. (1988). Prenatal Management of 746 Pregnancies at Risk for Congenital Toxoplasmosis. New England Journal of Medicine, 318, $271-275$. http://dx.doi.org/10.1056/NEJM198802043180502

Derouin, F. (2001). Anti-Toxoplasmosis Drugs. Current Opinion: Investigative Drugs, 2, 1368-1374.

Desmonts, G., \& Couvreur, J. (1974). Congenital Toxoplasmosis-A Prospective Study of 378 Pregnancies. New England Journal of Medicine, 290, 1110-1116. http://dx.doi.org/10.1056/NEJM197405162902003

Dodgson, J. E., Garwick, A., Blozia, S. A., Patterson, J. M., Bennett, F. C., \& Blum, R. W. (2000). Uncertainty in Childhood Chronic Conditions and Family Distress in Families of Young Children. Journal of Family Nursing, 6, 252-266. 
http://dx.doi.org/10.1177/107484070000600304

Dunn, D., Wallon, M., Peyron, F., Peterson, E., Peckham, C., \& Gilbert, R. (1999). Mother-to-Child Transmission of Toxoplasmosis: Risk Estimates for Clinical Counselling. Lancet, 353, 1829-1833. http://dx.doi.org/10.1016/s0140-6736(98)08220-8

Eichenwald, H. F. (1960). A Study of Congenital Toxoplasmosis with Particular Emphasis on Clinical Manifestations, Sequellae and Therapy. In J. C. Siim (Ed.), Human Toxoplasmosis (pp. 41-49). Copenhagen: Munksgaard.

Foulon, W., Villena, I., Stray-Pedersen, B., Decaster, A., Lappalainen, M., Pinon, J. M. et al. (1999). Treatment of Toxoplasmosis during Pregnancy: A Multicenter Study of Impact on Fetal Transmission and Children's Sequelae at Age 1 Year. American Journal of Obstetrics and Gynecology, 180, 410-415. http://dx.doi.org/10.1016/S0002-9378(99)70224-3

Freeman, K., Salt, A., Prusa, A., Malm, G., Ferret, N., Buffolano, W. et al. (2005). Association between Congenital Toxoplasmosis and Parent-Reported Developmental Outcomes, Concerns, and Impairments, in 3 Year Old Children. BMC Pediatrics, 5, 23. http://dx.doi.org/10.1186/1471-2431-5-23

Gilbert, R. E., Gras, L., Wallon, M., Peyron, F., Ades, A. E., \& Dunn, D. T. (2001). Effect of Prenatal Treatment on Mother to Child Transmission of Toxoplasma gondii: Retrospective Cohort Study of 554 Mother-Child Pairs in Lyon, France. International Journal of Epidemiology, 30, 1303-1308. http://dx.doi.org/10.1093/ije/30.6.1303

Gioia, G. A., Isquith, P. K., Guy, S. C., \& Kenworthy, L. (2000). Behavior Rating Inventory of Executive Function Professional Manual. Lutz, FL: Psychological Assessment Resources.

Guerina, N. G., Hsu, H.-W., Meissner, H. C., Maguire, J. H., Lynfield, R., Stechenberg, B. et al. (1994). Neonatal Serologic Screening and Early Treatment for Congenital Toxoplasma gondii Infection. New England Journal of Medicine, 330, 1858-1863. http://dx.doi.org/10.1056/NEJM199406303302604

Hohlfeld, P., Daffos, F., Thulliez, P., Aufrant, C., Couvreur, J., MacAlees, J. et al. (1989). Fetal Toxoplasmosis: Outcome of Pregnancy and Infant Follow-Up after in Utero Treatment. Journal of Pediatrics, 115, 765-769. http://dx.doi.org/10.1016/S0022-3476(89)80660-2

Jeremy, R. J., Kim, S., Nozyce, M., Nachman, S., McIntosh, K., Pelton, S. I. et al. (2005). Neuropsychological Functioning and Viral Load in Stable Antiretroviral Therapy-Experienced HIV-Infected Children. Pediatrics, 115, 380-387.

http://dx.doi.org/10.1542/peds.2004-1108

Katz, S. (2002). When the Child's Illness Is Life Threatening: Impact on the Parents. Pediatric Nursing, 28, 453-463.

Koppe, J. G., Loewer-Sieger, D. H., \& de Roever-Bonnet, H. (1986). Results of 20-Year Follow-Up of Congenital Toxoplasmosis. Lancet, 1, 254-256. http://dx.doi.org/10.1016/S0140-6736(86)90785-3

Korkman, M., Kirk, U., \& Kemp, S. (1998). NEPSY: A Developmental Neuropsychological Assessment Manual. San Antonio, TX: The Psychological Corporation.

Manly, T., Robertson, I. H., Anderson, V., \& Nimmo-Smith, I. (1999). The Test of Everyday Attention for Children Manual. Bury St. Edmunds: Thames Valley Test Co Ltd.

Mather, N., \& Woodcock, R. W. (2001). Woodcock-Johnson III Tests of Achievement Examiner's Manual. Itasca, IL: Riverside Publishing.

McAuley, J., Boyer, K. M., Patel, D., Mets, M., Swisher, C., Roizen, N. et al. (1994). Early and Longitudinal Evaluations of Treated Infants and Children and Untreated Historical Patients with Congenital Toxoplasmosis: The Chicago Collaborative Treatment Trial. Clinical Infectious Disease, 18, 38-72. http://dx.doi.org/10.1093/clinids/18.1.38

McGee, T., Wolters, C., Stein, L., Kraus, N., Johnson, D., Boyer, K. et al. (1992). Absence of Sensorineural Hearing Loss in Treated Infants and Children with Congenital Toxoplasmosis. Otolaryngology, Head and Neck Surgery, 106, 75-80.

McInerney, R. J., Hrabok, M., \& Kerns, K. A. (2005). The Children's Size-Ordering Task: A New Measure of Nonverbal Working Memory. Journal of Clinical and Experimental Neuropsychology, 27, 735-745.

http://dx.doi.org/10.1081/13803390490918633

McLeod, R., Boyer, K., Karrison, T., Kasza, K., Swisher, C., Roizen, N. et al. (2006). Outcome of Treatment for Congenital Toxoplasmosis, 1981-2004: The National Collaborative Chicago-Based, Congenital Toxoplasmosis Study. Clinical Infectious Disease, 42, 1383-1394. http://dx.doi.org/10.1086/501360

Mets, M. B., Holfels, E., Boyer, K. M., Swisher, C. N., Roizen, N., Stein, L. et al. (1996). Eye Manifestations of Congenital Toxoplasmosis. American Journal of Ophthalmology, 122, 309-324.

Meyers, J. E., \& Meyers, K. R. (1995). Rey Complex Figure Test and Recognition Trial: Professional Manual. Odessa, FL: Psychological Assessment Resources.

Michel, J. A., Kerns, K. A., \& Mateer, C. A. (2005). The Effect of Reinforcement Variables on Inhibition in Children with ADHD. Child Neuropsychology, 11, 295-302. http://dx.doi.org/10.1080/092970490911270

Patel, D. V., Holfels, E. M., Vogel, N. P., Boyer, K. M., Mets, M. B., Swisher, C. N. et al. (1996). Resolution of Intracranial Calcifications in Infants with Treated Congenital Toxoplasmosis. Radiology, 199, 433-440. 
Peyron, F., Wallon, M., \& Bernardoux, C. (1996). Long-Term Follow-Up of Patients with Congenital Ocular Toxoplasmosis. New England Journal of Medicine, 334, 993-994. http://dx.doi.org/10.1056/NEJM199604113341517

Remington, J. S., McLeod, R., Thulliez, P., \& Desmonts, G. (2006). Toxoplasmosis. In J. S. Remington, J. O. Klein, C. B. Baker, \& C. J. Wilson (Eds.), Infectious Diseases of the Fetus and Newborn Infant (6th ed., pp. 947-1091). Philadelphia, PA: Elsevier Saunders. http://dx.doi.org/10.1016/B0-72-160537-0/50033-5

Roberts, J., Mortimer, T., Mish, S., Kerns, K., Jagdis, F., \& MacMath, S. (2005). The Experiences and Recommendations of Families with Children Who Have Congenital Toxoplasmosis. Physical Disabilities: Education and Related Services, 24, 23-39.

Roizen, N., Swisher, C. N., Stein, M. A., Hopkins, J., Boyer, K., Holfels, E. et al. (1995). Neurologic and Developmental Outcome in Treated Congenital Toxoplasmosis. Pediatrics, 95, 11-20.

Sheslow, D., \& Adams, W. (1990). Wide Range Assessment of Memory and Learning Administration Manual. Wilmington, DE: Jastak Associates.

Wallon, M., Kodjikian, L., Binquet, C., Gerweg, J., Fleury, J., Quantin, C., \& Peyron, F. (2004). Long-Term Ocular Prognosis in 327 Children with Congenital Toxoplasmosis. Pediatrics, 113, 1567-1572. http://dx.doi.org/10.1542/peds.113.6.1567

Wechsler, D. (1991). Wechsler Intelligence Scale for Children-Third Edition Manual. San Antonio, TX: The Psychological Corporation.

Wilson, C. B., Remington, J. S., Stagno, S., \& Reynolds, D. W. (1980). Development of Adverse Sequelae in Children Born with Subclinical Congenital Toxoplasma Infection. Pediatrics, 66, 767-774.

\section{Appendix}

Table A1. List of neuropsychological, academic, and behavioral outcome measures.

1) Wechsler Intelligence Scale for Children-Third Edition (WISC-III) (Wechsler, 1991).

2) Woodcock-Johnson III Tests of Achievement (WJ-III ACH) (Mather \& Woodcock, 2001).

3) A Developmental Neuropsychological Assessment (NEPSY) (Korkman, Kirk, \& Kemp, 1998)—Comprehension of Instructions, Design Copying, Design Fluency, Manual Motor Sequencing, Phonological Processing, Verbal Fluency, Visual Attention, \& Visuomotor Precision.

4) Grooved Pegboard Test.

5) Rey Complex Figure Test (RCFT) (Meyers \& Meyers, 1995).

6) Conners’ Continuous Performance Test (CPT) (Conners, 1994).

7) Test of Everyday Attention for Children (TEA-Ch) (Manly, Robertson, Anderson, \& Nimmo-Smith, 1999)—Code Transmission, Creature Counting, Opposite Worlds, \& Walk, Don't Walk.

8) Wide Range Assessment of Memory and Learning (WRAML) (Sheslow \& Adams, 1990)—Design Memory, Picture Memory, Sentence Memory, Story Memory, Verbal Learning, \& Visual Learning.

9) Fire Fighter Game (Michel, Kerns, \& Mateer, 2005).

10) Children’s Size-Ordering Task (CSOT) (McInerney, Hrabok, \& Kerns, 2005).

11) Behavior Rating Inventory of Executive Function (BRIEF)—Parent Form (Gioia, Isquith, Guy, \& Kenworthy, 2000).

12) Conners' Parent Rating Scale-Revised: Long Version (Conners, 1997).

13) Scales of Independent Behavior—Revised (SIB-R) (Bruininks, Woodcock, \& Weatherman, 1996). 